\title{
Organization of Intracortical Circuits in Relation to Direction Preference Maps in Ferret Visual Cortex
}

\author{
B. Roerig ${ }^{1}$ and J. P. Y. Kao ${ }^{2}$ \\ ${ }^{1}$ Department of Anatomy and Neurobiology and ${ }^{2}$ Medical Biotechnology Center and Department of Physiology, University \\ of Maryland School of Medicine, Baltimore, Maryland 21201
}

Neurons in the primary visual cortex are selective for the direction of movement of a visual stimulus. Like other stimulus features, direction preference is mapped on the cortical surface in a systematic manner. Intracortical synaptic circuits, in particular inhibitory connections, have been implicated in the emergence of direction selectivity. Whether intracortical inhibition specifically suppresses responses to the nonpreferred direction or has a nonspecific "thresholding" effect is still controversial. To address these questions we investigated the relationship between patterns of intracortical synaptic connections and direction domains in ferret primary visual cortex (area
17) using a combined in vivo-in vitro approach. Excitatory synaptic inputs were iso-direction-tuned. The majority of local inhibitory inputs were also iso-direction-tuned. However, 40\% of inhibitory connections originated in regions preferring the opposite direction. These findings indicate that specific inhibitory interactions between cortical regions of opposite direction preference may contribute to the emergence and sharpening of direction selectivity.

Key words: visual cortex; direction selectivity; optical imaging; photostimulation; intracortical circuits; inhibition
Direction selectivity is a primary feature of cortical neurons required for the analysis of object motion (Hubel and Wiesel, 1962). Direction preference is organized in a columnar fashion (Tolhurst et al., 1981; Berman et al., 1987) and systematically mapped on the cortical surface (Weliky et al., 1996; Shmuel and Grinvald, 1996). The mechanisms underlying direction selectivity, in particular the role of intracortical inhibition, remain controversial.

A number of different models have been proposed: (1) inhibition is broadly tuned or untuned and acts in a nonselective manner by controlling the firing threshold of cells and thereby enhancing a directional bias present in the excitatory input ("iceberg' effect"; Hammond and Kim, 1996; Jagadeesh et al., 1997); (2) directionality is created by direction-specific lateral inhibition (Creuzfeldt et al., 1974; Goodwin et al., 1975; Bishop et al., 1980; Sillito, 1984; Eysel et al., 1988; Livingstone, 1998); 3) direction selectivity is a result of the spatiotemporal receptive field structure (Reid et al., 1991; De Valois and Cottaris, 1998), which in turn depends on intracortical inhibition (Murthy and Humphrey, 1999); and (4) reciprocal inhibition between pairs of GABAergic interneurons in layer 4 combined with temporal low-pass filtering (spike threshold) by cortical neurons accounts for direction selectivity (Maex and Orban, 1991). Modeling studies are also controversial. In some models isotropic circular inhibition is sufficient to create a directional bias (Woergoetter et al., 1992), whereas in others spatially asymmetric intracortical inhibition is required to create direction selectivity (Ruff et al., 1987; Sabatini and Solari, 1999).

Here, we have characterized the relationship between the intrinsic synaptic organization and the spatial representation of

\footnotetext{
Received July 26, 1999; revised Oct. 5, 1999; accepted Oct, 11, 1999.

We thank Gladys L. Ngatchou and Esther E. Kim for excellent technical assistance.

Correspondence should be addressed to Dr. Birgit Roerig, Department of Anatomy and Neurobiology, Medical School, University of Maryland at Baltimore, 685 West Baltimore Street, Baltimore, MD 21201-1509. E-mail: broer001@ umaryland.edu.

Copyright (ㄷ) 1999 Society for Neuroscience $\quad 0270-6474 / 99 / 190001-\bullet \$ 05.00 / 0$
}

direction preference on the cortical surface to determine whether synaptic inputs to a single cortical neuron are nonselective or originate preferentially from domains preferring the same or the opposite direction of motion. Our data indicate that excitatory connections are iso-direction-tuned, in line with a role of intrinsic excitatory circuits in amplifying direction-specific responses. In contrast, a significant proportion of inhibitory inputs originates from cortical regions of opposite direction preference, indicating that specific inhibitory mechanisms may contribute to direction tuning of cortical neurons.

\section{MATERIALS AND METHODS}

Ferrets [postnatal day 37 (P37)-P48; Marshall Farms, New Rose, NY] were anesthetized and prepared for in vivo optical imaging as previously described (Weliky et al., 1995, 1996). The cortex was illuminated with red light $(707 \mathrm{~nm})$. A $50 \times 50$ tandem lens combination and a Peltier-cooled slow-scan CCD camera (Optical Imaging Europe, Martinsried-Munich, Germany) was used for optical imaging. Visual stimulation was provided monocularly through the contralateral eye. Visual stimuli were presented at a distance of $30 \mathrm{~cm}$. Animals were presented with a dot pattern $(1.5 \times$ $1.5^{\circ}$ dot size) moving in different directions at a velocity of $15 \mathrm{deg} / \mathrm{sec}$ interleaved with blank screen presentations. The average dot density was 2.2 dots per $10 \times 10^{\circ}$ region. Four to eight directions were imaged per animal; stimuli were presented in a randomly interleaved manner. Singlecondition responses (averages of 120-180 trials) were divided by images

This article is published in The Journal of Neuroscience, Rapid Communications Section, which publishes brief, peerreviewed papers online, not in print. Rapid Communications are posted online approximately one month earlier than they would appear if printed. They are listed in the Table of Contents of the next open issue of JNeurosci. Cite this article as: JNeurosci, 1999, 19:RC44 (1-5). The publication date is the date of posting online at www.jneurosci.org.

http://www.jneurosci.org/cgi/content/full/3759 
acquired during blank screen presentations. The single-condition images were vector-summed to produce an angle map of direction or orientation preference (Bonhoeffer and Grinvald, 1993).

Intracortical injections of rhodamine-conjugated latex microspheres (70-150 nl) were made to guide alignment of in vivo and in vitro maps. The imaged cortex was then removed, and tangential slices $(400 \mu \mathrm{m}$ thickness) were prepared. Recordings were made at $33^{\circ} \mathrm{C}$ in a temperature-controlled recording chamber mounted on the stage of an upright microscope (BX50W I; Olympus Optical, Tokyo, Japan). Fluorescent bead marks were viewed using epifluorescence and a rhodamine filter set (Olympus). Slice overview images were taken using a CCD camera (DAGE-MTI, Michigan City, IN) and a Snappy (Play Incorporated, Rancho Cordova, CA) video frame acquisition module. Whole-cell patch-clamp recordings were done at holding potentials of -60 and -20 $\mathrm{mV}$ to distinguish between excitatory and inhibitory synaptic inputs (Katz and Dalva, 1994).

Synaptic inputs were scanned by local photolysis of Nmoc-caged glutamate (1 mm; Rossi et al., 1997) (see Katz and Dalva, 1994 for detailed description of scanning laser photostimulation). An argon-krypton ion laser (Stabilite 2017; Spectra-Physics, Fremont, CA) was used as a UV light source. The laser beam was coupled into a $50-\mu \mathrm{m}$-diameter fiber optic attached to a motorized $x / y$ stage. The fiber was moved within an oil droplet below the recording chamber. The laser beam was manually focused into the middle of the slice preparation. Opening of the external shutter, scanning of the laser beam, and data acquisition were controlled by a National Instruments (Austin, TX) analog-to-digital board (AT$\mathrm{MIO} / \mathrm{AI}$ E-10) and custom software (Labview, National Instruments). The flash duration was $5-10 \mathrm{msec}$, and the interstimulus interval was 3-5 sec. The spacing of stimulation sites was $50 \mu \mathrm{m}$. The number of points mapped per neuron ranged from 560 to 950 , corresponding to an area of $\sim 1.5-2.5 \mathrm{~mm}^{2}$. Postsynaptic cells were filled with biocytin (1\%), and labeled cells were visualized by standard immunoperoxidase staining techniques.

Alignment of direction maps obtained in vivo and synaptic input ("photostimulation") maps were guided by the fluorescent bead injections. Optical imaging maps, video images of slice preparations, photostimulation maps, and histological sections were overlayed using the layer menu of Adobe (Mountain View, CA) Photoshop. Linear scaling and rotation were applied to the images until the bead marks were at least $50 \%$ overlapping. The bead marks were $100-200 \mu \mathrm{m}$ in diameter, which results in a maximum alignment error of $75 \mu \mathrm{m}$. This corresponds to an orientation preference error of $<10^{\circ}$ (Weliky et al., 1995). Synaptic input maps were then superimposed on the direction map. For each site giving rise to a synaptic input as well as for the location of the postsynaptic cell, the orientation value was calculated as the mean of four pixels. The direction-tuning differences between the location of postsynaptic neurons and the sites of origin of synaptic inputs were determined.

\section{RESULTS}

Data included in this study were obtained from a total of 15 layer $2 / 3$ pyramidal neurons from seven animals. Figure 1 shows examples of typical recordings. The total number of EPSCs analyzed was 447 from $n=15$ neurons; the total number of IPSCs was 279 recorded from $n=13$ neurons. EPSC amplitudes ranged from 8 to $76 \mathrm{pA}$; IPSC amplitudes ranged from 15 to $89 \mathrm{pA}$. Local events tended to be larger than long-range inputs. The direction-tuning differences between the location of the postsynaptic cell and all its inputs were determined. Direction preference ranges from 0 to $360^{\circ}$. The direction preference of each presynaptic site can deviate from the postsynaptic site by a value between -180 and + $180^{\circ}$. We have not observed asymmetries in direction-tuning differences. The tuning histograms shown in Figure 2 are therefore rectified, i.e., showing only absolute tuning differences. For subsequent statistical tests, events were binned in $20^{\circ}$ categories (0-20 to $160-180^{\circ}$ direction-tuning difference; Fig. $\left.2 A-C\right)$.

\section{Excitatory intracortical connections link neurons preferring the same direction of stimulus motion}

The majority of synaptic inputs $(76 \%)$ were evoked from sites in a distance of $<1 \mathrm{~mm}$ from the recording site. The majority of
A B
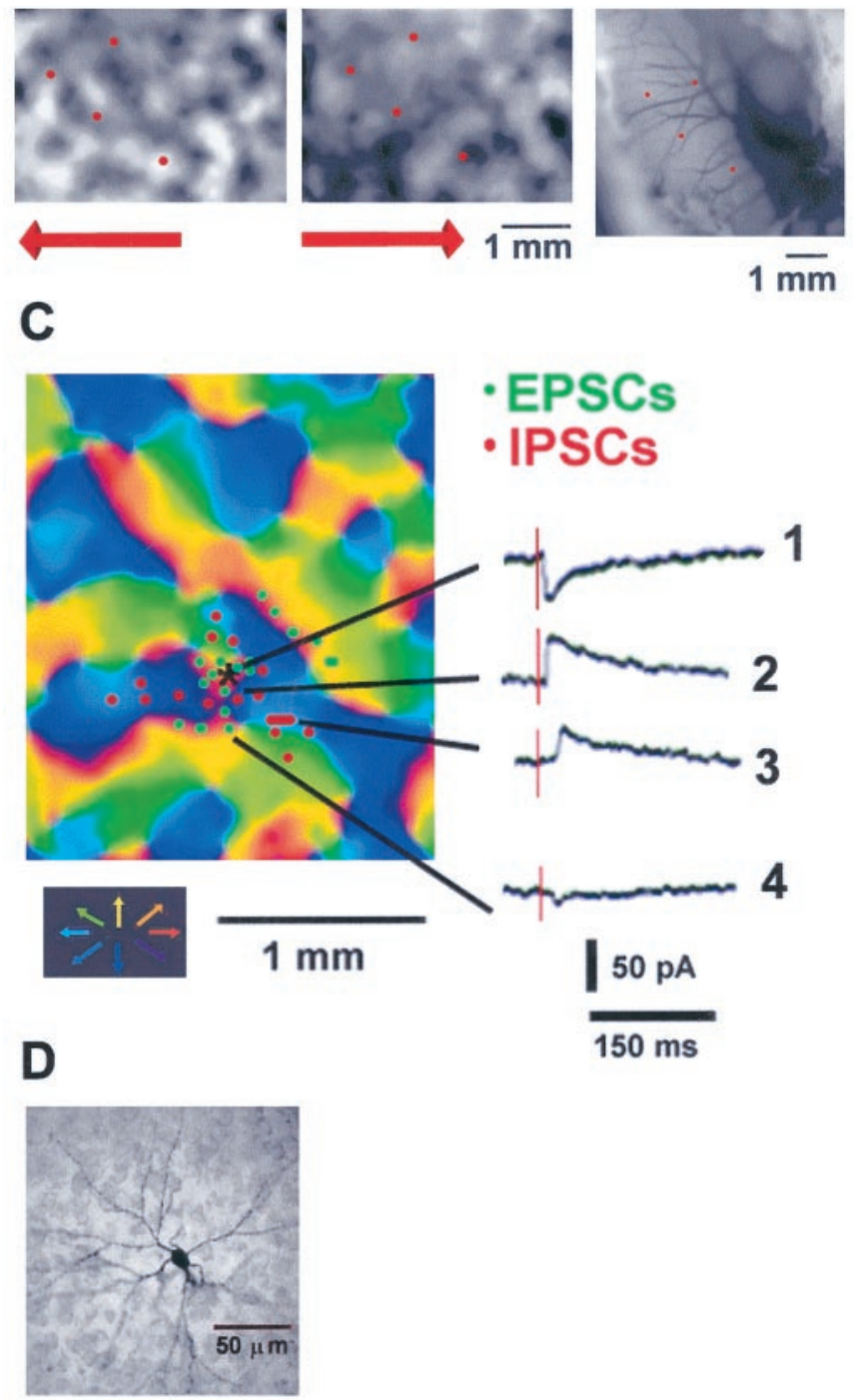

Figure 1. A, Examples of single-condition images. Arrows indicate direction of motion of the stimulus. $B$, Blood vessel reference image (age of animal, P42). Red dots in $A$ and $B$ represent the sites of alignment bead injections. $C$, Location of postsynaptic neuron (black star) and sites providing excitatory (red dots) and inhibitory (green dots) synaptic inputs superimposed on direction polar map. Insets, Photostimulation-evoked responses (1, 4, EPSCs; 2, 3, IPSCs). D, Postsynaptic neuron intracellularly filled with biocytin in a tangential slice prepared from the imaged cortex.

EPSCs originated from regions of similar direction preference compared with the location of the postsynaptic neuron $\left(<60^{\circ}\right.$ direction-tuning difference; Fig. 2). On average, iso-direction tuning was more prominent in local (originating within $600 \mu \mathrm{m}$ distance from the postsynaptic cell) than in long-range excitatory connections (Fig. 3A).

\section{Inhibitory inputs can originate in regions preferring the same or the opposite direction}

The majority of inhibitory inputs also originated from locations of similar direction preference, but a significant proportion (37.8\%) of IPSCs originated in regions preferring the opposite direction compared with the recording site (Fig. 2). This indicates a contribution of specific inhibitory connections to direction tuning. 
A
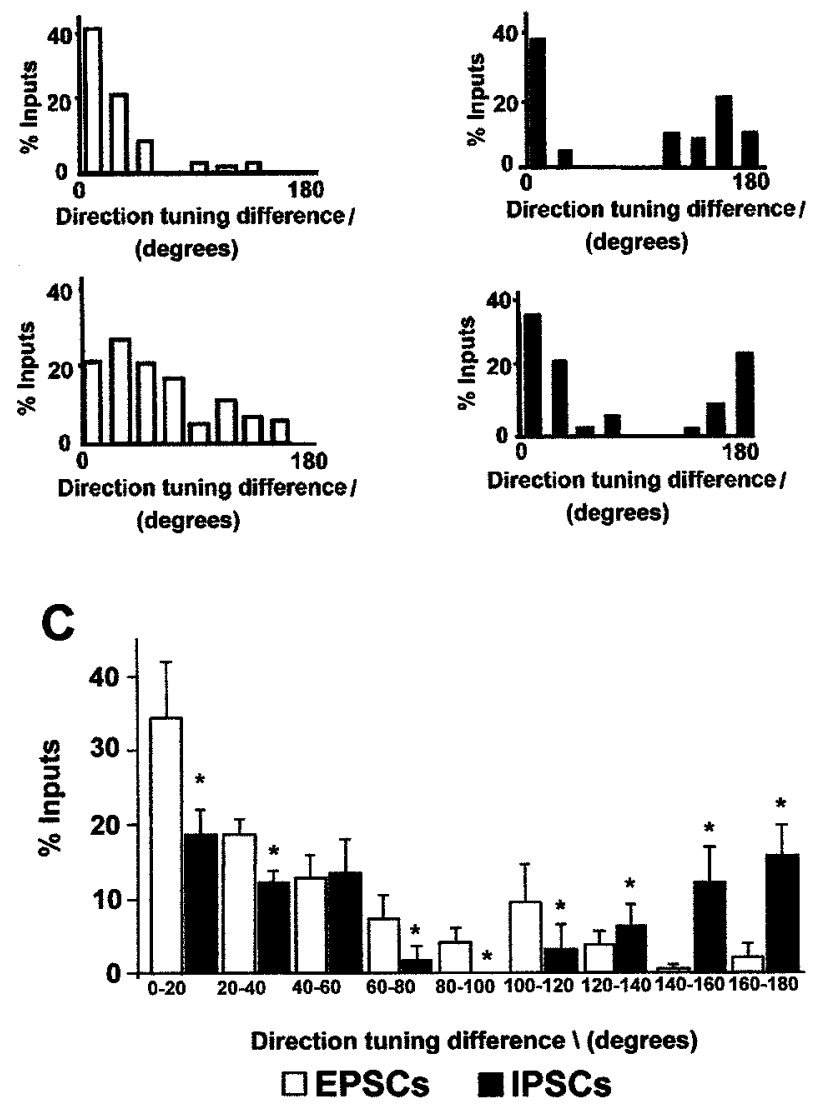

D

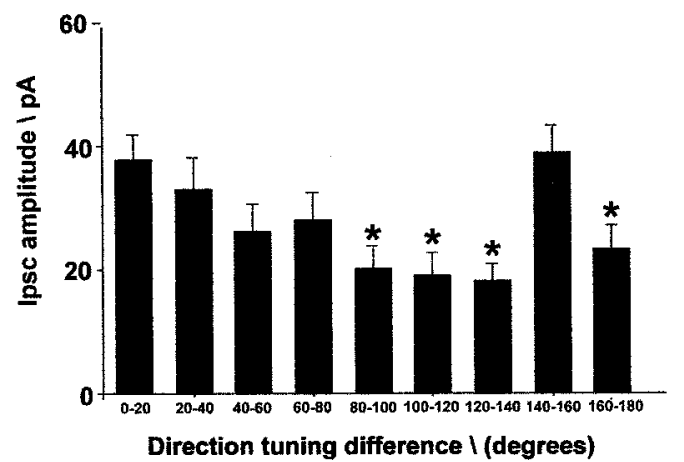

Figure 2. $A, B$, Tuning histograms of synaptic inputs to two layer $2 / 3$ pyramidal neurons. The percentage of total synaptic inputs is plotted against the direction-tuning difference between presynaptic and postsynaptic sites. $A$, Excitatory inputs preferentially originate in cortical regions preferring the same direction of stimulus motion. $B$, The direction-tuning histograms of IPSCs show two peaks: inhibitory inputs originate from regions preferring the same and from regions preferring the opposite direction. $C$, Direction-tuning histogram pooled from $n=13$ layer $2 / 3$ pyramidal neurons. The bars in figure $C$ represent the avarage number of inputs falling into each direction-tuning difference category from a total of 13 neurons. Error bars represent SEM of number of inputs showing indicated direction-tuning difference averaged among the total population of cells. The first white bar (EPSCs) and the first black bar (IPSCs) represent the same range of direction-tuning difference (i.e., $0-20^{\circ}$ ), each following pair of white and black bars represent the same tuning difference range. *Statistically significant differences of percentages of EPSCs and IPSCs falling into each category. $D$, Mean IPSC amplitude plotted against direction-tuning difference. Error bars represent SEM. *Statistically significant differences compared with the $0-20^{\circ}$ category.
A
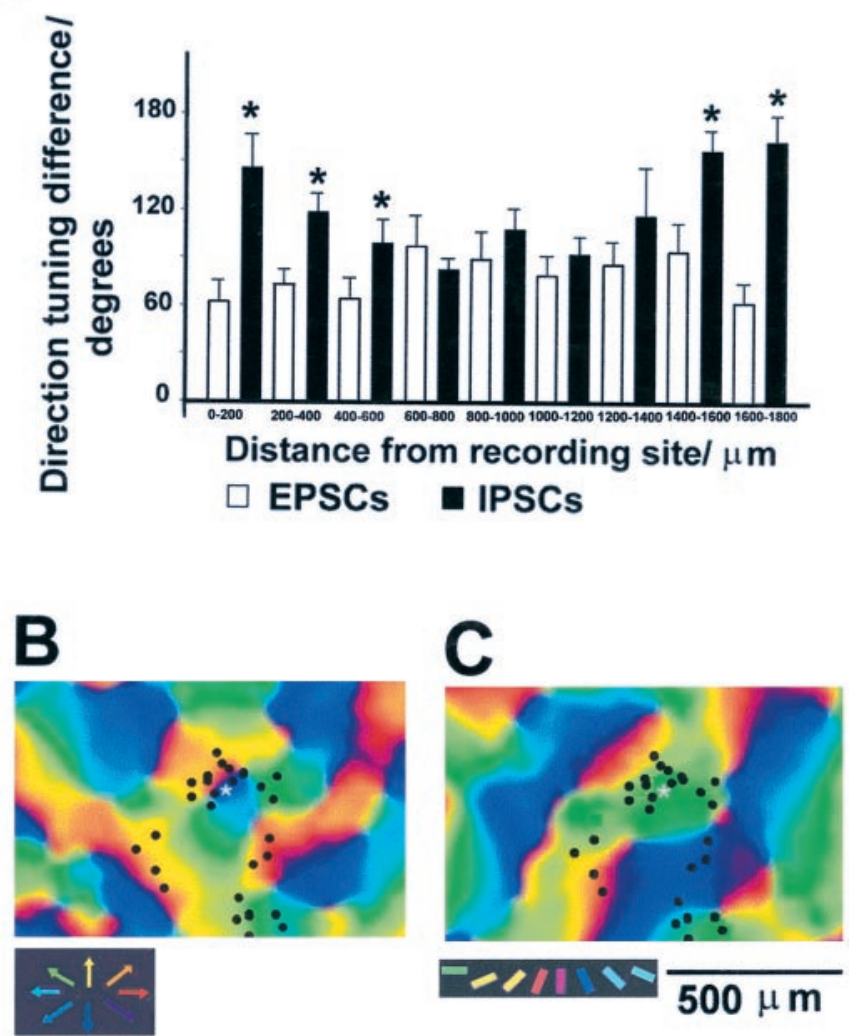

Figure 3. A, Direction-tuning difference between presynaptic and postsynaptic cells plotted against distance between recording and stimulation sites. The tuning difference as well as the $x$ - and $y$-distance values from the recording site for each site giving rise to a synaptic input have been determined. The actual distance between presynaptic and postsynaptic sites has been calculated using Pythagorean arithmetic. $A$, Synaptic events have been pooled according to distance categories, and the mean tuning difference for each distance category has been determined. The bars represent the average direction-tuning difference of all synaptic events recorded from all 13 neurons in a given distance range. The error bars represent the SEM of average direction-tuning difference in each indicated distance range. Adjacent white (EPSCs) and black (IPSCs) bars represent the same distance range. Excitatory inputs mostly originated from regions preferring the same direction of stimulus motion as the recorded neuron throughout the entire distance range sampled. In contrast, local and remote inhibitory inputs originated in regions preferring opposite directions. * Significant differences between the average tuning of EPSCs and IPSCs falling into each distance category. $B, C$, Examples of inhibitory input patterns superimposed on the direction map $(B)$ and the orientation map recorded from the same animal $(C)$. Black dots, Locations of origins of inhibitory inputs; white star, location of postsynaptic cell. A large number of local inputs originate from regions preferring the opposite direction compared with the postsynaptic cell, whereas orientation preference is similar for presynaptic and postsynaptic sites.

The distributions of EPSCs and IPSCs have been compared for $n=13$ individual neurons. The number of inhibitory inputs in $n=2$ cells was $<10$ events; these neurons have not been included in the analysis. The percentages of EPSCs and IPSCs falling into each category $\left(0-20\right.$ to $160-180^{\circ}$ direction-tuning difference) have been calculated for each individual neuron. The percentages of events in each category have then been statistically compared among the 13 layer 2/3 neurons (Mann-Whitney rank sum test). Except for the $40-60^{\circ}$ category, the percentages of EPSCs and IPSCs were significantly different in each bin $(p=<0.001$; Fig. $2 C)$. Next we determined the mean amplitude of inhibitory syn- 
aptic inputs (IPSCs) for every direction-tuning difference category (Fig. 2D). We have statistically compared the event amplitudes in the $0-20^{\circ}$ bin, i.e., the events showing the strongest iso-direction tuning, with all the other categories using one-way ANOVA. The median values for the 160-180, 120-140, and $100-140^{\circ}$ tuning difference categories were significantly smaller than the median of the $0-20^{\circ}$ bin. However, there was no difference in mean amplitude of the events in the $140-160^{\circ}$ category compared with iso-direction-tuned events. Thus, there is variability of IPSC strength and a tendency for events tuned to opposite directions to be smaller.

In conclusion, the intracortical organization of inhibitory connections in relation to direction domains is different from the excitatory connection scheme. Inhibitory input distributions were not flat but showed two peaks at both the same and the opposite directions (Fig. 2), indicating different mechanisms involving inhibition tuned to either the preferred or the nonpreferred direction.

\section{Spatial relationship between synaptic input patterns and direction and orientation maps}

Iso-orientation domains in ferret and cat visual cortex tend to be subdivided into two direction domains preferring opposite directions of stimulus motion (Shmuel and Grinvald, 1996; Weliky et al., 1996). The inhibitory inputs tuned to the opposite direction may thus originate locally, from the subregion of the isoorientation domain preferring the opposite direction, or from long-range inhibitory connections as described in cat visual cortex (Eysel et al., 1988). We next analyzed the direction tuning of EPSCs and IPSCs as a function of distance between presynaptic and postsynaptic sites (Fig. 3A). Within 0-600 $\mu \mathrm{m}$ distance between recording sites and stimulation sites the direction-tuning difference between presynaptic and postsynaptic sites was significantly larger for inhibitory inputs than for excitatory inputs ( $p=$ $<0.001$, Mann-Whitney rank sum test). In $n=3$ animals the orientation preference map was also recorded. In these cases we have overlayed the synaptic input map, the direction map, and the orientation map. In five neurons analyzed we observed a clear tendency for local inhibitory inputs to originate from subdomains of opposite direction preference within an iso-orientation domain (Fig. 3B,C). However, the remote inhibitory inputs also tended to be tuned to the opposite direction. In a distance of 1.4-2 mm between stimulation and recording sites the tuning of IPSCs again significantly differed from the tuning of EPSCs $(p=<$ 0.001, Mann-Whitney rank sum test), indicating that some longrange inhibitory connections also specifically target regions preferring the opposite direction of stimulus motion.

\section{DISCUSSION}

The relationship of intracortical synaptic circuits and orientation and ocular dominance maps has been addressed in a number of anatomical studies (Gilbert and Wiesel, 1989, Malach et al., 1993; Bosking et al., 1997). Comparatively little is known about the organization of intrinsic circuits and direction preference maps. Here, we have analyzed the relationship between intracortical synaptic connections and direction domains to elucidate how intrinsic circuitry contributes to the creation and spatial representation of direction preference.

\section{Iso-direction tuning of excitatory intracortical connections: amplification by recurrent excitation?}

Local and long-range excitatory synaptic connections link neurons of similar orientation preference (Nelson and Frost, 1985; Tso et al., 1986; Gilbert and Wiesel. 1989; Bosking et al., 1997; Roerig and
Katz, 1998). A similar organizational principle appears to hold true for direction preference. Local photostimulation-evoked EPSCs were iso-direction-tuned. This is in line with a role for local excitatory circuits in amplifying direction-specific responses (Suarez et al., 1995). Long-range connections were on average less direction-specific than local connections. On the level of individual neurons they tended to be either sharply orientation- or directiontuned; i.e., one or the other stimulus parameter seems to dominate the specificity of horizontal connections. Thus, on the one hand there is a tendency for long-range connections to link regions of similar direction preference but different retinotopic position, similar to the proposed role for orientation-specific lateral connectivity in feature binding (Singer and Gray, 1995). On the other hand, the lack of absolute specificity for both orientation and direction in a given neuron may reflect the necessity of intracortical circuits to compromise some of their selectivity to accommodate multiple maps of stimulus dimensions.

\section{Inhibitory inputs tuned to the preferred and opposite directions: implications for mechanisms of direction selectivity}

The majority of inhibitory synaptic connections originated locally, within $500 \mu \mathrm{m}$ distance between recording and stimulation sites. This is in line with the predominantly local distribution of axonal arbors of cortical interneurons (Kisvarday et al., 1985; Bealieu and Somogyi, 1990). A significant fraction of inhibitory inputs $(37.8 \%)$ originated in sites deviating $>90^{\circ}$ in direction tuning from recorded neurons. Indeed, $18 \%$ of IPSCs showed a tuning difference of $160-180^{\circ}$ compared with the recording site; i.e., they were sharply tuned for the opposite direction of motion. These inputs were partially local, partially long-range in origin. Basket cells with axonal projections extending for $>1 \mathrm{~mm}$ have been described (Martin et al., 1983). Although the total output of these basket cells is not orientation- or direction-specific (Kisvarday et al., 1994), individual axonal clusters are confined to a single functional domain (Buzas et al., 1997), indicating that these neurons can be recruited in multiple ways and probably contribute to a number of different receptive field properties. Large basket cells are thus a major candidate for the long-range inhibitory inputs observed in the present study.

It has been known for a long time that intracortical inhibitory connections contribute to the generation and sharpening of direction tuning in the primary visual cortex (for review, see Sillito, 1984), yet the precise mechanisms remain controversial. Selective inhibition-suppressing responses to the nonpreferred direction have been reported in cat (Creutzfeld et al., 1974; Eysel et al., 1988; Crook et al., 1996, 1997) and monkey (Livingston, 1998) visual cortex. Our data are in line with these findings, inasmuch as we find a significant proportion of inhibitory inputs to a single cortical neuron originating in regions tuned to the nonpreferred direction.

Recent in vivo studies, on the other hand, indicate that both excitatory and inhibitory visually evoked postsynaptic potentials are tuned to the preferred direction (Douglas et al., 1991; Jagadeesh et al., 1997). Our results are to some extent also in agreement with these findings, because the majority of intracortical EPSCs and IPSCs were iso-direction-tuned. However, we do find inhibitory inputs tuned to both the preferred and the nonpreferred directions in the same neuron. The two types of inputs are thus unlikely to correspond to different classes of cortical neurons. Direction selectivity may thus involve iso-directiontuned inhibitory inputs, which threshold out untuned excitatory 
inputs, and, on top of this mechanism, inhibitory inputs tuned to the nonpreferred direction may provide further refinement.

\section{Inhibitory mechanisms in orientation and direction tuning: different mechanisms?}

In a previous study we investigated the relationship between orientation maps and intrinsic circuits in different layers of ferret visual cortex (Roerig and Katz, 1998). We have found no evidence for cross-orientation inhibition. The tuning of inhibitory inputs was broader than the tuning of excitatory inputs in a subpopulation of cells, but in general EPSPs and IPSCs were iso-orientation tuned. The organization of inhibitory circuits in relation to direction maps appears different; in addition to isodirection-tuned inhibitory inputs, we were able to demonstrate a population of inputs tuned to the nonpreferred direction. Thus, a recurrent mechanism (Somers et al., 1995) may be sufficient to generate orientation tuning, whereas direction specificity appears to require more selective mechanisms. Directionality is to a larger extent dependent on temporal aspects such as response timing and velocity (Duysens et al., 1987). Moreover, direction selectivity is more susceptible to removal of inhibition than orientation selectivity (Sillito 1984), in line with different mechanisms involved in the two properties. In conclusion, a combination of specific and nonselective mechanisms may be required to set up the different receptive field properties of cortical neurons.

\section{REFERENCES}

Bealieu C, Somogyi P (1990) Targets and quantitative distribution of GABAergic synapses in the visual cortex of the cat. Eur $\mathbf{J}$ Neurosci 2:296-303.

Berman NE, Wilkes ME, Payne BR (1987) Organization of orientation and direction selectivity in areas 17 and 18 of cat cerebral cortex. J Neurophysiol 58:676-699.

Bishop PO, Kato H, Orban GA (1980) Direction selective cells in complex family in cat striate cortex. J Neurophysiol 43:1266-1283.

Bonhoeffer T, Grinvald A (1993) The layout of iso-orientation domains in area 18 of cat visual cortex: optical imaging reveals a pinwheel like organization. J Neurosci 13:4157-4180.

Bosking WH, Zhang Y, Schofield B, Fitzpatrick D (1997) Orientation selectivity and the arrangement of horizontal connections in tree shrew striate cortex. J Neurosci 17:2112-2127.

Buzas P, Eysel UT, Kisvarday ZF (1997) Functional topography of basket cells revealed with optical imaging in the visual cortex of the cat. Soc Neurosci Abstr 23:1029.

Creutzfeld OD, Kuhnt U, Benevento LA (1974) An intracellular analysis of visual cortical neurones to moving stimuli: responses in a cooperative neuronal network. Exp Brain Res 21:251-274.

Crook JM, Kisvarday ZF, Eysel UT (1996) GABA-induced inactivation of functionally characterized sites in cat visual cortex (area 18): effects on direction selectivity. J Neurophysiol 75:2071-2088.

Crook JM, Kisvarday ZF, Eysel UT (1997) GABA-induced inactivation of functionally characterized sites in cat striate cortex: effects on orientation tuning and direction selectivity. Vis Neurosci 14:141-158.

De Valois RL, Cottaris NP (1998) Inputs to directionally selective simple cells in macaque striate cortex. Proc Natl Acad Sci USA 95:14488-14493.

Douglas RD, Martin KAC, Whitteridge D (1991) An intracellular analysis of the visual responses of neurones in cat visual cortex. J Physiol (Lond) 440:659-696.

Duysens J, Maes H, Orban GA (1987) The velocity dependence of direction selectivity of visual cortical neurons. J Physiol (Lond) 387:95-113.

Eysel UT, Muche T, Woergoetter F (1988) Lateral interactions at direction selective striate neurons in the cat demonstrated by local cortical inactivation. J Physiol (Lond) 399:657-675.

Ganz L (1984) Visual cortical mechanisms responsible for direction selectivity. Vision Res 24:3-11.

Gilbert CD, Wiesel TN (1989) Columnar specificity of intrinsic horizontal and corticocortical connections in cat visual cortex. J Neurosci 9:2432-2442.

Goodwin AW, Henry GH, Bishop PO (1975) Direction selectivity of simple striate cells: properties and mechanism. J Neurophysiol 38:1500-1523.
Hammond P, Kim J-N (1996) Role of suppression in shaping orientation and direction selectivity of complex neurons in cat striate cortex. J Neurophysiol 75:1163-1176.

Hubel DH, Wiesel, TN (1962) Receptive fields, binocular interaction and functional architecture in the cat's visual cortex. J Physiol (Lond) 160:106-154.

Jagadeesh B, Wheat HS, Kontsevich LL, Tyler CW, Ferster D (1997) Direction selectivity of synaptic potentials in simple cells of the cat visual cortex. J Neurophysiol 76:2772-2789.

Katz LC, Dalva MB (1994) Scanning photostimulation: a new approach for analyzing brain circuits. J Neurosci Methods 54:205-218.

Kisvarday ZF, Martin KAC, Whitteridge D, Somogyi P (1985) Synaptic connections of intracellularly filled clutch cells: a type of small basket cells in the visual cortex of the cat. J Comp Neurol 241:111-137.

Kisvarday ZF, Kim DS, Eysel UT, Bonhoeffer T (1994) Relationship between lateral inhibitory connections and the topography of the orientation map in cat visual cortex. Eur J Neurosci 10:1619-1632.

Livingstone MS (1998) Mechanisms of direction selectivity in macaque V1. Neuron 20:509-526.

Maex R, Orban GA (1991) Subtraction inhibition combined with a spiking threshold accounts for cortical direction selectivity. Proc Natl Acad Sci USA 88:3549-3553.

Malach R, Amir Y, Harel M, Grinvald A (1993) Relationship between intrinsic connections and functional architecture revealed by optical imaging and in vivo targeted biocytin injections in primate striate cortex. Proc Natl Acad Sci USA 90:10469-10473.

Martin KAC, Somogyi P, Whitteridge D (1983) Physiological and morphological properties of identified basket cells in the cat's visual cortex. Exp Brain Res 50:193-200.

Murthy A, Humphrey AL (1999) Inhibitory contributions to spatiotemporal receptive-field structure and direction selectivity in simple cells of cat area 17. Am J Physiol 1212-1224.

Nelson JI, Frost BJ (1985) Intracortical facilitation among co-oriented, coaxially aligned simple cells in cat striate cortex. Exp Brain Res 61:54-61.

Reid RC, Soodak RE, Shapley RM (1991) Directional selectivity and spatiotemporal structure of receptive fields of simple cells in cat striate cortex. J Neurophysiol 66:505-529.

Roerig B, Katz LC (1998) Relationship between synaptic input patterns and orientation preference maps in ferret visual cortex. Soc Neurosci Abstr 24:765.

Rossi FM, Margulis M, Tang C-M, Kao JPY (1997) N-Nmoc-Lglutamate, a new caged glutamate with high chemical stability and low pre-photolysis activity. J Biol Chem 272:32933-32939.

Ruff RT, Rauschecker JP Guenther P (1987) A model for direction selective "simple" cells in the visual cortex based on inhibition asymmetry. Biol Cybern 57:147-157.

Sabatini SP, Solari F (1999) An architectural hypothesis for direction selectivity in the visual cortex: a role for spatially asymmetric intracortical inhibition. Biol Cybern 80:171-183.

Shmuel A, Grinvald A (1996) Functional organization for direction of motion and its relationship to orientation maps in cat area 18. J Neurosci 16:6945-6964.

Sillito AM (1984) Functional considerations of the operation of GABAergic inhibitory processes in the visual cortex. In: Cerebral cortex, Vol 2 (Jones EG, Peters A, eds), pp 91-117. New York: Plenum.

Singer W, Gray CM (1995) Visual feature integration and the temporal correlation hypothesis. Annu Rev Neurosci 18:555-586.

Somers DC, Nelson SB, Sur M (1995) An emergent model of orientation selectivity in cat visual cortical simple cells. J Neurosci 15:5448-5465.

Suarez H, Koch C, Douglas R (1995) Modeling direction selectivity of simple cells in striate visual cortex within the framework of the canonical microcircuit. J Neurosci 15:6700-6719.

Tolhurst DJ, Dean AF, Thompson ID (1981) Preferred direction ofmovement as an element in the organization of cat visual cortex. Exp Brain Res 44:340-342.

Ts'o DY, Gilbert CD, Wiesel TN (1986) Relationship between horizontal interactions and functional architecture in cat striate cortex as revealed by cross-correlation analysis. J Neurosci 6:1160-1170.

Weliky M, Kandler K, Fitzpatrick D, Katz LC (1995) Patterns of excitation and inhibition evoked by horizontal connections in visual cortex share a common relationship to orientation columns. Neuron 16:541-552.

Weliky M, Bosking WH, Fitzpatrick D (1996) A systematic map of direction preference in primary visual cortex. Nature 379:725-728.

Woergoetter F, Niebur E, Koch C (1992) Generation of direction selectivity by isotropic intracortical connections. Neural Comput 4:332-340. 